\title{
Attention aux bordures !
}

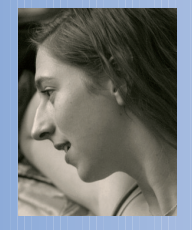

Lise MAUDUA DELVA

Docteur en chirurgie dentaire,

$3 \mathrm{~b}$ Breestraat,

2311CG Leiden, Pays-Bas.

\section{Robert CAVEZIAN}

Cabinet d'imagerie dentaire Echelle, Saint-Honoré,

Paris.

\section{Gérard PASQUET}

Cabinet d'imagerie dentaire Echelle, Saint-Honoré,

Paris.
«N'omettez jamais d'examiner attentivement les bordures de vos clichés.»

A. Djian

Cette femme de 50 ans consulte pour bilan pré-implantaire dans le secteur de 35. Elle est en bonne santé.,

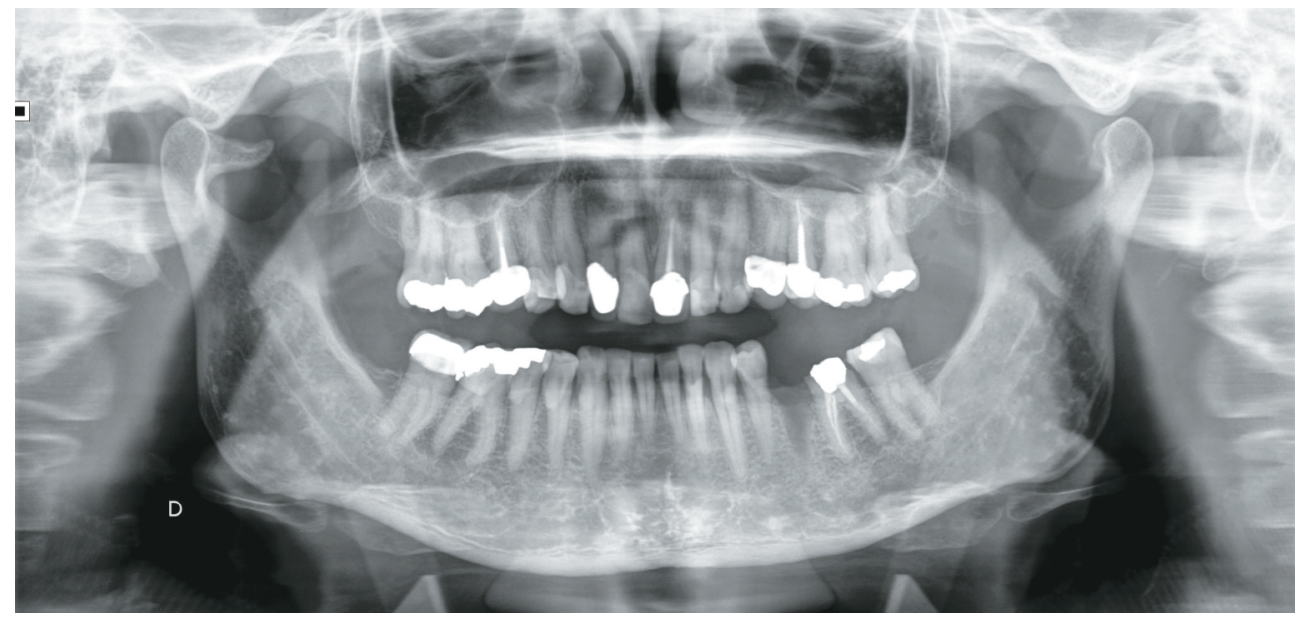

AOS 2009;247:203-205

DOI:10.1051/aos/2009012

(C) AEOS / EDP Sciences 


\section{A. Topographie}

\section{$>$ Que notez-vous?}

a. Une lacune dans le secteur des apex de 12 et 11.

b. Une altération de la morphologie d'un condyle mandibulaire.

c. Des processus coronoïdes longs.

d. Des angles mandibulaires marqués.

\section{B. Morphologie,} densité, structure

\section{> Quelle description sémiologique} correspond le mieux à l'anomalie relevée?

a. Amincissement du col du condyle mandibulaire droit.

b. Ostéophyte marginal antérieur du col du condyle mandibulaire droit, déformation de la tête condylaire et ostéocondensation sous-chondrale.

c. Image grossièrement ovalaire à grand axe antéro-postérieur de densité calcique et de structure homogène se projetant entre la face antérieure du condyle mandibulaire et le processus coronoïde droit.

d. Processus occupant l'espace de la face antérieure du condyle mandibulaire droit, triangulaire à grand axe antéro-postérieur à base condylaire de densité corticale périphérique et de densité médullaire centrale aux contours réguliers et bien limités.

e. Image grossièrement ovalaire à grand axe horizontal développée autour des extrémités apicales des racines de 12 et 11 , de densité liquidienne et de structure homogène.

\section{Conclusion}

> Quel est votre diagnostic ?

a. Ostéome du condyle mandibulaire droit.

b. Kyste apico-dentaire sur 12 et 11.

c. Exostose du col du condyle mandibulaire droit.

d. Myosite ossifiante.

e. Arthropathie dégénérative de l'ATM droite.

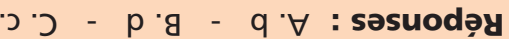

\section{Commentaires}

Image caractéristique : corticale osseuse en parfaite continuité avec la corticale de l'os porteur et spongieuse en parfaite continuité avec la spongieuse de l'os porteur.

L'image périapicale sur 12 et 11 est une image construite par la radioclarté de la fossette latérale et la radio opacité de l'aile du nez.

\section{Points à retenir}

Exostose solitaire ou ostéochondrome : tumeur osseuse bénigne parmi les plus fréquentes [1]. Excroissance osseuse développée à la surface de la zone métaphysaire d'un os à ossification enchondrale, recouverte d'une coiffe cartilagineuse, sessile ou pédiculée $[2,4]$. 
Il convient de distinguer l'exostose ostéogénique solitaire (fortuite et unique), des exostoses ostéogéniques multiples ou maladie exostosante : héréditaire, mode de transmission autosomique dominant, gènes identifiés : EXT 1, 2 et 3 [1].

Le diagnostic de l'exostose ostéogénique solitaire est radiologique.

Radiographie standard: typiquement perpendiculaire à la métaphyse, sessile ou pédiculée. L'os médullaire et le cortex de la tumeur sont en continuité avec ceux de l'os porteur [5].

\section{Complications}

Risque de dégénerescence maligne (chondrosarcome) chez l'adulte uniquement dans le cas de la maladie exostosante, 5 à 10 p. 100 des cas [3].

Fracture sur exostose pédiculée.

Déformations osseuses.

Liées à l'effet de masse de l'exostose : compressions vasculaires, compressions médullaires et radiculaires, compressions locales, troubles de l'articulé dentaire dans le cas d'une exostose mandibulaire.

\section{Bibliographie}

1. Maroteaux P, Le Merrer M Maladies osseuses de l'enfant (4e éd). Paris : Médecine sciences Flammarion, 2002.

2. Cavezian $\mathrm{R}$, Pasquet $\mathrm{G}$, Bel G, Baller G. Imagerie dento-maxillaire (deuxième édition).

Paris : Masson, 2001.

3. Nahum $\mathrm{H}$ et al. Traité d'imagerie médicale. Paris, Médecine sciences Flammarion, 2004.

4. Railhac JJ et al. Imagerie des tumeurs osseuses.

Paris : Sauramps médical, 1999.

5. Chotel F, Goin F. Tumeurs osseuses bénignes. Cahier d'enseignement de la SOFCOT. Elsevier, 2005. 


\section{LA COMMUNICATION ET Y'EUCATION DU PATIENT}

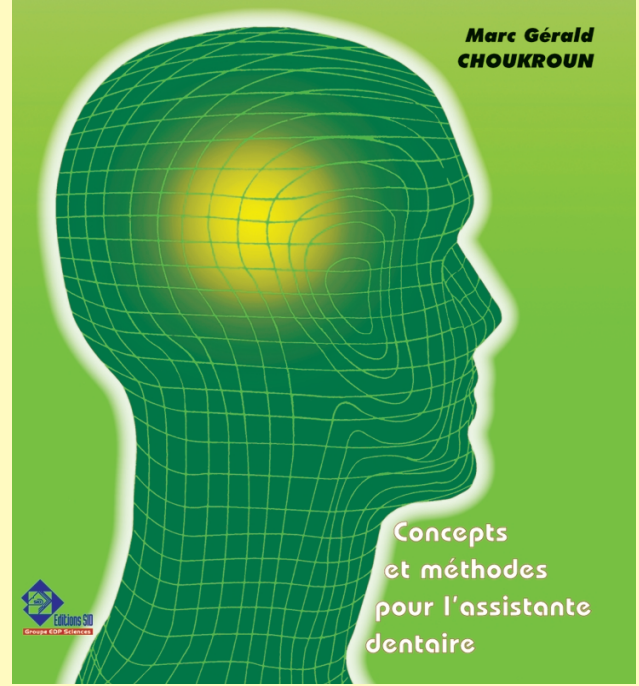

\section{LA COMMUNICATION ET LÉDUCATION DU PATIENT}

Marc Gérald CHOUKROUN

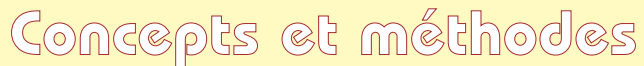

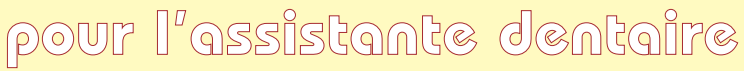

Reliure souple format $17 \times 24 \mathrm{~cm}-88$ pages

PRIX : $23 €$ (frais de port : $8 €$ )

L e premier contact qui sétablit entre un patient et le cabinet den-

$L_{\text {taire passe systématiquement par lintermédiaire de l'assistante }}$ dentaire. Que ce soit par la réponse téléphonique ou à l'accueil son rôle est de toute évidence prépondérant.

Il s'agit entre l'assistante et le patient d'un acte de communiquant.

En un mot tout passe par la communication, par le relationnel.

L'auteur est convaincu que le secret d'une bonne relation entre soigné et soignant repose sur la maitrise de six dimensions qui sont :

- La technique bio-médicale ;

- L'information ;

- L'éducation ;

- Le transfert ;

- L'environnement psycho-social ;

- L'encadrement.

Le souhait de l'auteur est que le lecteur ou la lectrice trouve dans cet ouvrage des idées concrètes débouchant sur des changements de comportement.

L'objectif est de permettre à l'assistante de mieux gérer son stress et se sentir en accord avec elle-même sur le plan humain.

Son deuxième objectif est que les praticiens bénéficient d'une assistante qui ne se perde pas dans des relations humaines difficiles.

Son troisième objectif est que les patients aient le sentiment d'être compris, écoutés et qu'ils traduisent par leur coopération l'idée qu'ils bénéficient d'un soin de qualité.

\section{B ON DE COMMANDE à retourner à la Société d'Information et de Diffusion (SID) www.editionsid.com}

LA COMMUNICATION ET LÉDUCATION DU PATIENT - Marc Gérald CHOUKROUN

Nom :

Prénom :

Adresse :

Code Postal

Ville

Tél

Fax

E-mail.

Paiement effectué par chèque à l'ordre de : Société d'Information et de Diffusion (SID) - 9, rue Christine, 75006 PARIS Tél. + 33 (0)1 43293101 - Fax + 33 (0)1 43293262 - E-mail : sidedition@orange.fr

Paiement par carte bancaire : $\square$ Carte Visa

Master Card

$\mathrm{N}^{\circ}$

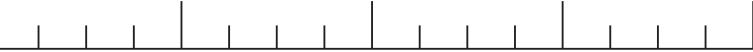

Date d'expiration

Signature obligatoire

Noter ici les 3 derniers chiffres situés au dos de la carte 